\title{
Simulation of the Radio Communication Channel with Unmanned Aerial Vehicle in Urban Conditions
}

\author{
Gennady Kulikov ${ }^{1, *}$, Alexander Nesterov², Andrey Leluh ${ }^{1,3}$ \\ ${ }^{1}$ Institute of Radio-Technical and Telecommunication Systems, Russian Technological University, Moscow, Russia \\ ${ }^{2}$ Automatics Concern, Joint-stock Company, Moscow, Russia \\ ${ }^{3}$ Moscow Scientific Research Institute of Radio Communication, Moscow, Russia
}

Email address:

kulikov@mirea.ru (G. Kulikov), nester980@yandex.ru (A. Nesterov), leluh@mniirs.org (A. Leluh)

${ }^{*}$ Corresponding author

\section{To cite this article:}

Gennady Kulikov, Alexander Nesterov, Andrey Leluh. Simulation of the Radio Communication Channel with Unmanned Aerial Vehicle in Urban Conditions. Journal of Electrical and Electronic Engineering. Vol. 6, No. 4, 2018, pp. 104-110. doi: 10.11648/j.jeee.20180604.11

Received: July 31, 2018; Accepted: August 23, 2018; Published: October 13, 2018

\begin{abstract}
Modern unmanned aerial vehicles (UAVs) are used in many spheres of human activity. In conditions of strong urban development, the radio communication channel with UAV is multi-path, which leads to a significant decrease in the quality of the transfer of useful information. Ensuring reliable and qualitative communication depends on the correct choice of the frequency range and the type of modulation used. Multiposition signals of M-PSK, M-QAM, M-FSK formats are considered. Due to the complexity of the mathematical description of such a radio channel, a computer simulation method was used to obtain the results. A multipath propagation model with a number of beams up to 10 is used. The dependencies of the probability of erroneous reception of information on the parameters of the communication channel are obtained: the signal-tonoise ratio, the number and intensity of the propagation paths. The energy parameters of the radio channel are calculated and the optimal frequency range is estimated from the maximum signal-to-noise ratio. It is indicated that the best in this criterion is the operating frequency range of $2-5 \mathrm{GHz}$. Calculations showed that the transmission coefficient of the radio channel is unstable and varies greatly in frequency. When using narrow-band signals M-PSK, M-QAM, this can lead to a situation where the signal can get into the "bad" frequency band and the quality of information transfer will decrease significantly. In connection with this, a radio channel with multiposition frequency modulation M-FSK was studied, when the transmission of discrete information is carried out using multiple parcels with different frequencies falling into both "bad" and "good" frequency zones. The results of the study showed that the probability of BER error in some cases improves by times compared to M-PSK and M-QAM. This allows us to talk about the prospects for the M-FSK format. Considering the good performance of this format, further studies are planned in the direction of using M-FSK format signals and their prospective version of MCPFSK (multi-position frequency modulated signals with a continuous phase) in the considered radio channel with different modulation and positioning indices, in particular CPFSK with modulation indices of 0.5 and 0.75 , together with MIMO and OFDM systems. The obtained results can be used in the design of modern and promising UAV-to-ground communication systems.
\end{abstract}

Keywords: Unmanned Aerial Vehicles (UAV), Digital Communication Systems, Multipath Transmission, High Level of Urban Development

\section{Introduction}

Modern unmanned aerial vehicles (UAVs) have found wide application in many areas of human life. Initially intended for conducting combat operations and monitoring the enemy, they eventually began to be used for civilian tasks: cargo delivery, monitoring of the earth's surface and various industrial facilities such as factories and industrial enterprises. It is worth noting the successful use of UAVs by rescue services to search for missing persons in hard-to-reach areas [1]. However, despite a rather wide range of tasks to be solved, many technical problems remain unresolved. One 
such problem is the use of UAVs in cities and suburbs with hight-density development. Let us explain how these zones differ from others. Any city, regardless of the degree of development, have buildings of different number of storeys, streets, forest areas and possible inconstancy of the forms of the terrain relief. In this case, the radio signal received and transmitted by the UAV is reflected from various objects, their surfaces, undergoing damping, i.e., the communication channel is multipath. In addition, there are other devices in the city that use the frequency resource, industrial disturbances of various kinds, and also the presence of deliberate interference. All of the above impacts are destabilizing factors that lead to distortion of information in the process of reception and transmission and possible loss of control over the UAV.

Full characteristics of the electromagnetic waves propagation can be obtained by solving the Maxwell equations under certain conditions that reflect the physical characteristics of the objects that create the obstacles. Good practical results are obtained by taking into account the relief of the underlying surface on the basis of the HuygensKirchhoff physical principle. Since these calculations are difficult to perform, and in many cases there are no parameters necessary for this purpose, to obtain the propagation characteristics of signals, approximation methods that do not require the solution of complex equations are often used. An example of such an approach are the formulas obtained in the works of N. I. Bardin, G. Z. Rubin, P. N. Trifonov, Y. Okamura, M. Hata [2, 3]. Models of Okamura-Hata, COST231-Hata, Wolfiz, Hata-Davidson, Xia-Bertoni are known and widely used [4].

But many of the existing empirical models, although they fully describe the features of the radio channel under investigation, do not satisfy the requirements arising in the development of modern communication systems, in particular, do not take into account the effects arising from the movement of objects, as well as the complicated interference situation in the radio channel.

Many studies have been devoted to the researches of the quality of communication using unmanned aerial vehicles, which indicates the importance of this scientific direction [519]. The authors of these papers use various methods of analysis: mathematical calculations by formulas, computer simulation, the use of empirical data. The main results of the studies are energy parameters of the radio channel, its throughput, characteristics of noise immunity, recommendations on the use of prospective types of signals are given.

In this paper, based on the study of the listed methods, the energy parameters (signal-to-noise + interference ratio) of the
UAV-to-ground radio channel were calculated, the models of multipath signal propagation in various conditions, including for the most complicated case (under the hight-density urban development), were realized and investigated.

\section{Characteristics of the UAV Radio Communication Channel}

The model of the UAV-to-ground radio channel used in the paper consists of several stages (Figure 1). At the first stage of simulation, the positions of UAV and ground-based transceivers, as well as the boundaries of urban development, the nature of the terrain of the countryside are determined and the propagation path of the direct signal is formed. The second stage takes into account the trajectory of the signal reflected from the ground surface in accordance with the methods of simulation of the two-path propagation of radio waves. The signal reflected from the ground surface changes its parameters (amplitude fluctuations according to the Rayleigh law and the phase change of the signal according to a uniform law). The input of the land receiver receives the sum of signals propagating along the direct and reflected beam. At the third stage, the signal reflected from the ground surface is subjected to a similar separation into a direct beam and a beam reflected from the buildings. This process is repeated also at subsequent stages, and at each next stage the intensity of the reflected beam decreases. Three situations were studied in the paper: one-beam propagation only along a direct beam, two-beam propagation (direct and reflected beams), multipath propagation (direct and several reflected beams).

In order to take into account the influence of the interference situation, interference is added to the received signal, which can be either simple fluctuation noise or a combination of interfering effects specific to a particular radio channel, for example, impulse noise.

The coefficient $\mu$ is used in the model, which characterizes the level of the signal reflected from the underlying surface or surface of any objects (structures). Its value varied from 0 to 0.5 , which for low values corresponds to weakly reflecting scattering surfaces (grass, forest, etc.), and for large ones highly reflecting surfaces (asphalt of streets, walls of houses, etc.). In addition, the model contained a parameter characterizing the rate of change in characteristics (delay, amplitude and phase fluctuations) of a beam reflected from exterior objects in the form of the ratio of the amplitude and phase variation frequency to the information transfer rate. This allowed us to simulate situations that occur at different UAV speeds from 0 to $200 \mathrm{~km} / \mathrm{h}$. 


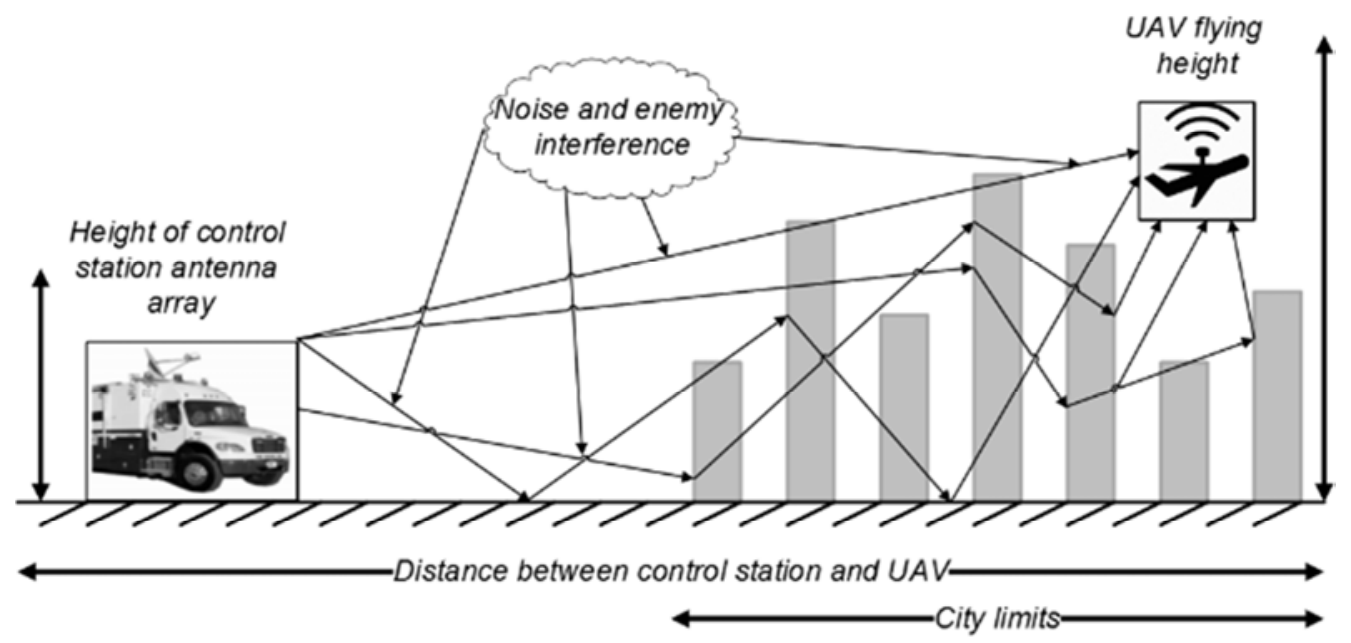

Figure 1. Model of signal propagation in urban conditions.

\section{Simulation Results}

In the simulation, the frequency dependences of the transmission ratio of the channel, $A(f)$, on the number of beams, $N$, and their intensity were investigated. The graphs characterizing these dependences are shown in Figure 2.

From the obtained relationships, it can be concluded that the transmission ratio is highly uneven and, as a result, the shape of the signal spectrum in the multipath channel is essential distorted. It is clear that in a real channel during UAV motion, this parameter will have a random character determined by the attenuation and amplification of the signal in different frequency segments.

The graphs for the error probability, BER, of the information symbol reception received during the simulation are shown in Figure 3 and 4. It was assumed that the transfer of discrete information is carried out using Binary PhaseShift Keying (BPSK). Figure 3 shows the case of a two-beam propagation (a direct and a reflected beams), in Figure 4 three-beam propagation.

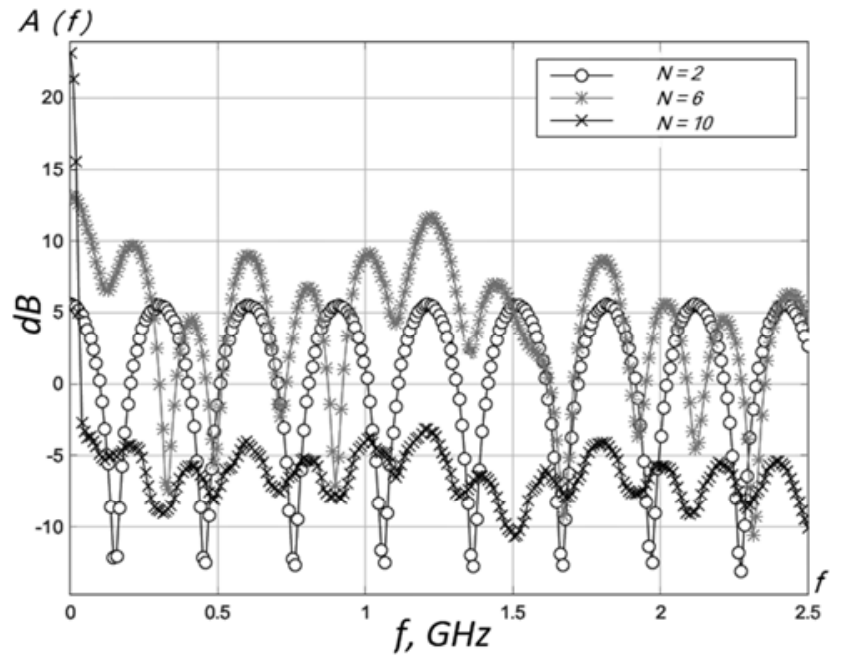

Figure 2. Variation of the channel transmission ratio with different number of beams in the communication channel.

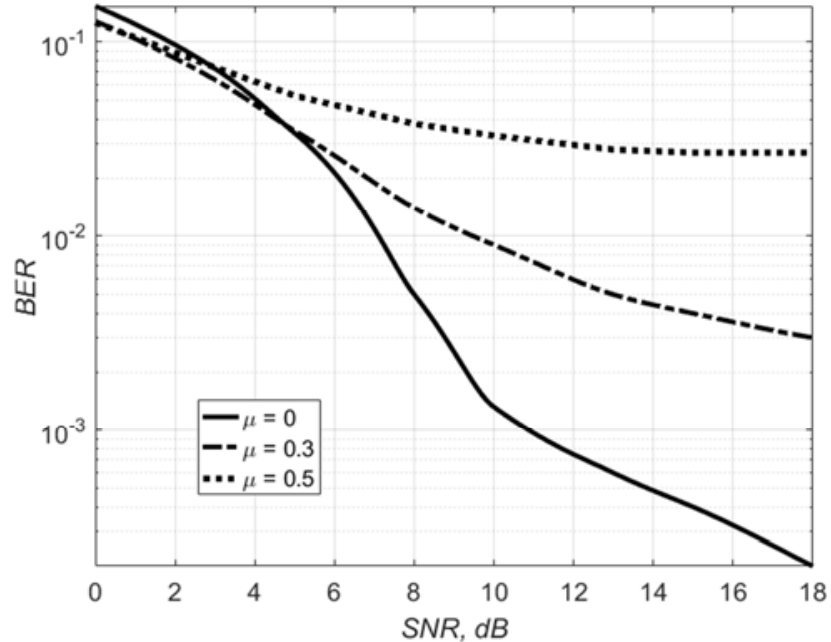

Figure 3. Relationship between the error probability, BER, and the signalto-noise ratio (in $\mathrm{dB}$ ) in a two-beam channel.

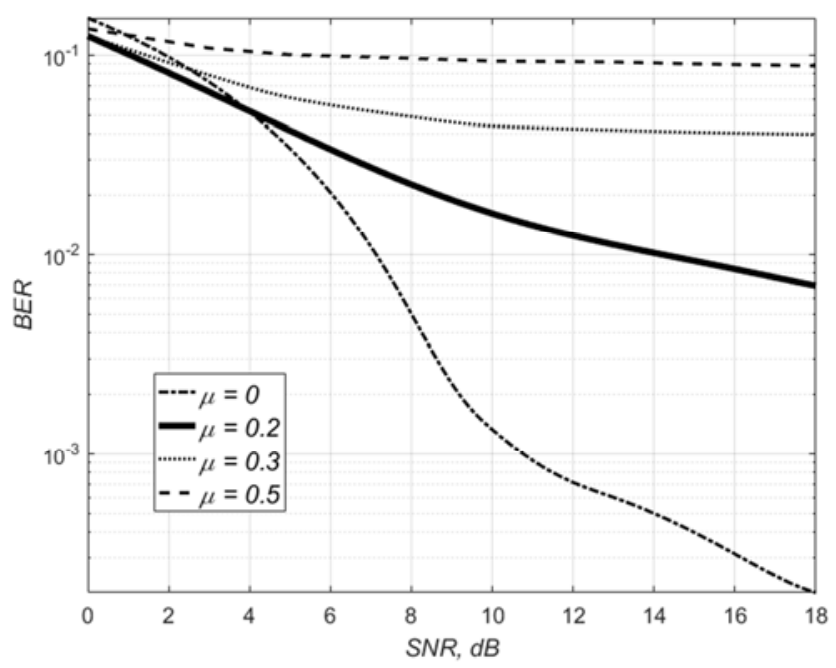

Figure 4. Relationship between the error probability, BER, and the signalto-noise ratio (in $\mathrm{dB}$ ) in a three-beam channel.

The results show a strong multipath effect on the signal reception quality. So, if the error probability decreases to $10^{-3}$ 
times with a two-beam and three-beam channel for small values of the $\mu$ coefficient, then for $\mu=0.5$ the error probability becomes unacceptably large (up to $10^{-1}$ ). The increase in the number of beams also strongly affects the reception quality. In particular, it can be argued that even at $\mu$ $=0.2$ in a three-beam channel, the error probability increases to $10^{-2}$, which is also unacceptable.

It is also established that the rate of fluctuations of the reflected beams determined by the relative mutual speed of the receiver and transmitter, practically does not affect the quality of signal reception in the studied range of the UAV speeds from 0 to $200 \mathrm{~km} / \mathrm{h}$.

The effect of industrial interference was simulated by the addition of a pulsed process with different repetition frequencies, $F$, and the Rayleigh amplitude distribution law. In this case, the parameter $F$ took values from $0.1 / T$ to $1 / T$, where $T$ is the duration of the information signal clock interval, and the normalized variance of the Rayleigh law is from 0.4 to 4 .

Depending on the combination of these parameters, the signal-to-interference ratio, $P_{s} / P_{\text {imp }}$, varied from 0.2 to 20 .

The complex situation, when all the distorting factors (multipath, additive noise, impulse noise) affect the radio channel, is shown in Figure 5.

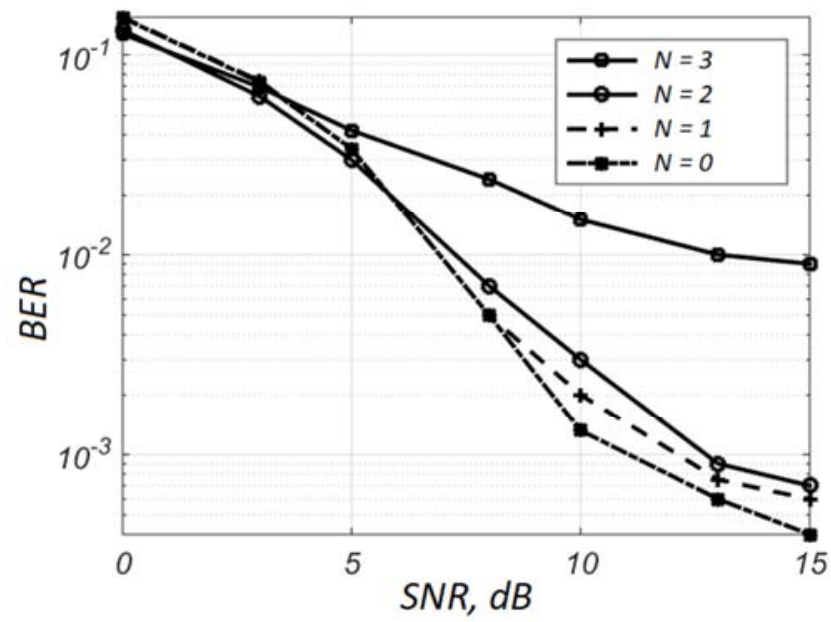

Figure 5. Influence of complex impact of interfering factors in a multipath radio channel.

It was found that the effect of pulsed interference is not as noticeable as the effect of multipath, and, at low intensity of such interference, it can be neglected. Its influence becomes noticeable at $P_{s} / P_{\text {imp }}<2$. Besides, the impact of such interference is notably reduced by well-known methods, such as the approach with a broadband amplifier, limiter and narrow-band filter connected consecutively.

The simulation of the influence of the Doppler effect showed that at a mutual speed of the receiver and the transmitter up to $200 \mathrm{~km} / \mathrm{h}$, no degradation of the noise immunity characteristics was recorded.

\subsection{Selecting the Optimal Frequency Range of the Channel}

Fidelity of the data transmission through the UAV to ground channel is essentially determined by the ratio of signal strength to the spectral density of noise strength, which depends on many factors and may be determined by the following equation:

The accuracy of data transmission over the UAV-to ground channel is substantially determined by the ratio of the signal power $P_{s}$ to the noise power spectral density $N_{0}$, which depends on a number of factors and can be determined from the following equation:

$$
\frac{P_{S}}{N_{0}}=\frac{P_{t} G(\gamma) \eta \lambda^{2}}{4 \pi d^{2} L_{a}(\gamma, H) K\left(T_{A}(\gamma, H) \eta+T_{0}\left(K_{n}-\eta\right)\right)}
$$

where $P_{t}$ is the transmitter power; $G(\gamma)$ is the gain of the antenna, which depends on the angle of the receiver with respect to thetransmitter $\gamma$ and the angle of the flight device in the direction of the ground, $\eta$ is the loss factor of the antenna-feeder path; $\lambda$ is the wavelength of the carrier signal; $d$ is the distance between the receiver and the transmitter; $L_{a}$ $(\gamma, H)$ is atmospheric losses, which depend on the elevation angle $\gamma$ and the altitude of the flight $H ; K$ is the Boltzmann constant; $T_{A}(\gamma, H)$ is noise temperature of the antenna, which depends on atmospheric noises and radio emission from space sources; $T_{0}$ is the absolute temperature of the medium $\left(290^{\circ} \mathrm{K}\right) ; K_{n}$ is noise factor of the receiver.

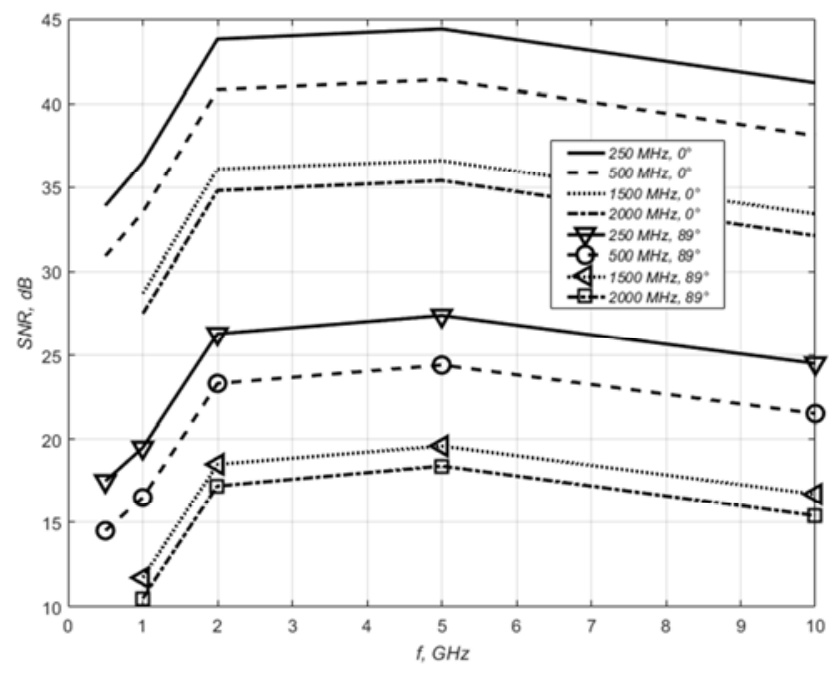

Figure 6. Relationships between the signal-to-noise ratio taking into account the industrial noise (in $\mathrm{dB}$ ) and the operating frequency for different receiver bandwidths $\triangle f$ and the UAV elevation angles $\gamma\left(0^{\circ}\right.$ and $\left.89^{\circ}\right)$.

Calculations show that in the absence of industrial noise with an increase in the operating frequency $f=c / \lambda$, the signalto-noise ratio decreases noticeably, although it remains large enough for reliable radio communication. For example, at $f=$ $2 \mathrm{GHz}$, the transmitter power is $10 \mathrm{~mW}$, the receiver band $\Delta f$ $=2 \mathrm{GHz}$, and the flight device distance more than $100 \mathrm{~km}$, the signal-to-noise ratio is $22 \mathrm{~dB}$, which, when using BPSK, gives an error probability of less than $10^{-7}$. At a frequency of $10 \mathrm{GHz}$, this ratio drops to $15 \mathrm{~dB}$. The situation changes significantly if we take into account the industrial noise characteristic for urban areas and which have a high intensity in the low-frequency ranges. Figure 6 shows the graphical 
relationships constructed from the calculated data obtained at UAV flight altitude $H=2 \mathrm{~km}$. Here, the values of signal-tonoise ratios are given taking into account industrial noise for different operating frequency ranges, the transmission bands of receivers $\Delta f$ with the receiver noise factor $K_{n}=4$ and different UAV elevation angles $\gamma$ (at an elevation angle $\gamma=0^{\circ}$, the distance between the receiver and the transmitter is $d=2$ $\mathrm{km}$, with $\gamma=89^{\circ}-114 \mathrm{~km}$ ).

A significant increase in the industrial noise levels with a decrease in operating frequency leads to a situation where the signal-to-noise ratio in the $0.5-1 \mathrm{GHz}$ bands is greatly reduced. In the range of 5-10 GHz, the levels of industrial noise are small, but with the increase in the operating frequency, the attenuation of the useful signal increases, and the resulting signal-to-noise ratio also decreases, although not so noticeably. Thus, the maximum signal-to-noise ratio falls within the range of $2-5 \mathrm{GHz}$, and this region is preferable for use in advanced UAV communication systems.

The effect of multipath propagation of radio waves worsens this situation. So, with a small number of beams and a small reflectivity of the surfaces $\mu=0.1-0.2$, we can expect a decrease in the signal-to-noise ratio by an additional $2-3$ $\mathrm{dB}$. With an increase in the number of beams and an increase in the reflection ratio of the surfaces $\mu$ up to $0.3-0.4$, the decrease in the signal-to-noise ratio can reach 10-15 dB. In this case, the useful signal can be completely masked by interference, therefore, in the construction of transceivers, it is necessary to provide for the development of methods for control multipath and methods of adaptive correction of the radio channel characteristics.

\subsection{Comments on the Selection of Modulation Type}

The quality of the communication in the radio channel is directly determined by the types of signals used and the information exchange protocols. There are currently no specific standards for the use of certain types of modulation in UAV radio communication systems. Currently, BPSK, Four-Phase Quadrature Amplitude Modulation (4-QAM) and 16-QAM formats are most commonly used in these systems [20]. The study and comparison of the noise immunity of a wider set of modulation types in the propagation of radio waves in the described medium is of interest.

The signal formats of Multiple Phase-Shift Keying (MPSK), Multiple Quadrature Amplitude Modulation (MQAM), and Multiple Frequency-Shift Keying (M-FSK) with various positional indices $\mathrm{M}$ were investigated. All the below simulation results were obtained with the following parameters: the UAV altitude $-2 \mathrm{~km}$, the ground station altitude $-3 \mathrm{~m}$, the UAV distance from the ground station $100 \mathrm{~km}$, the signal-to-noise ratio $E_{b} / N_{0}=7 \mathrm{~dB}$.

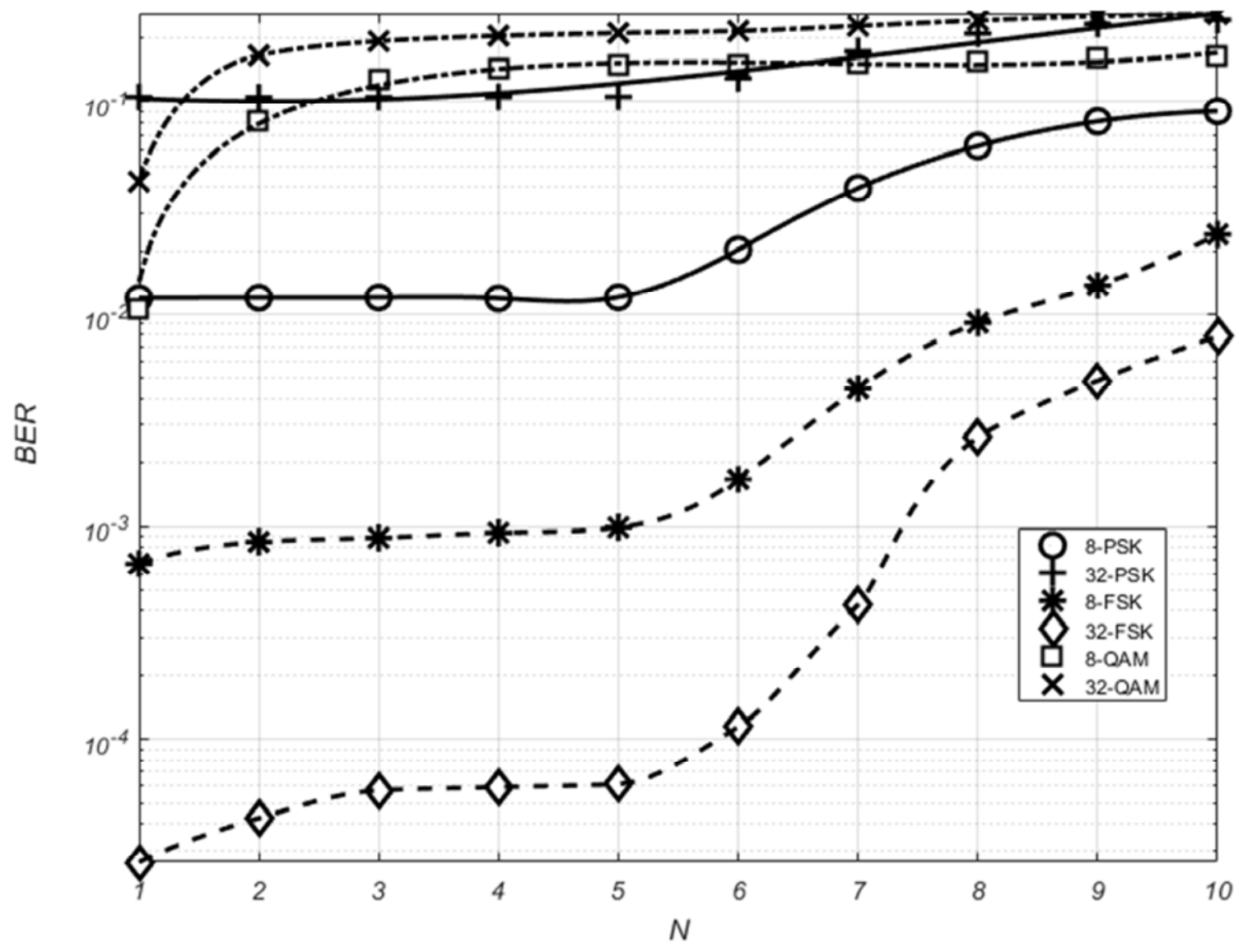

Figure 7. The relationship between the error probability, BER, and the number of beams, $N$, for different signal formats. 


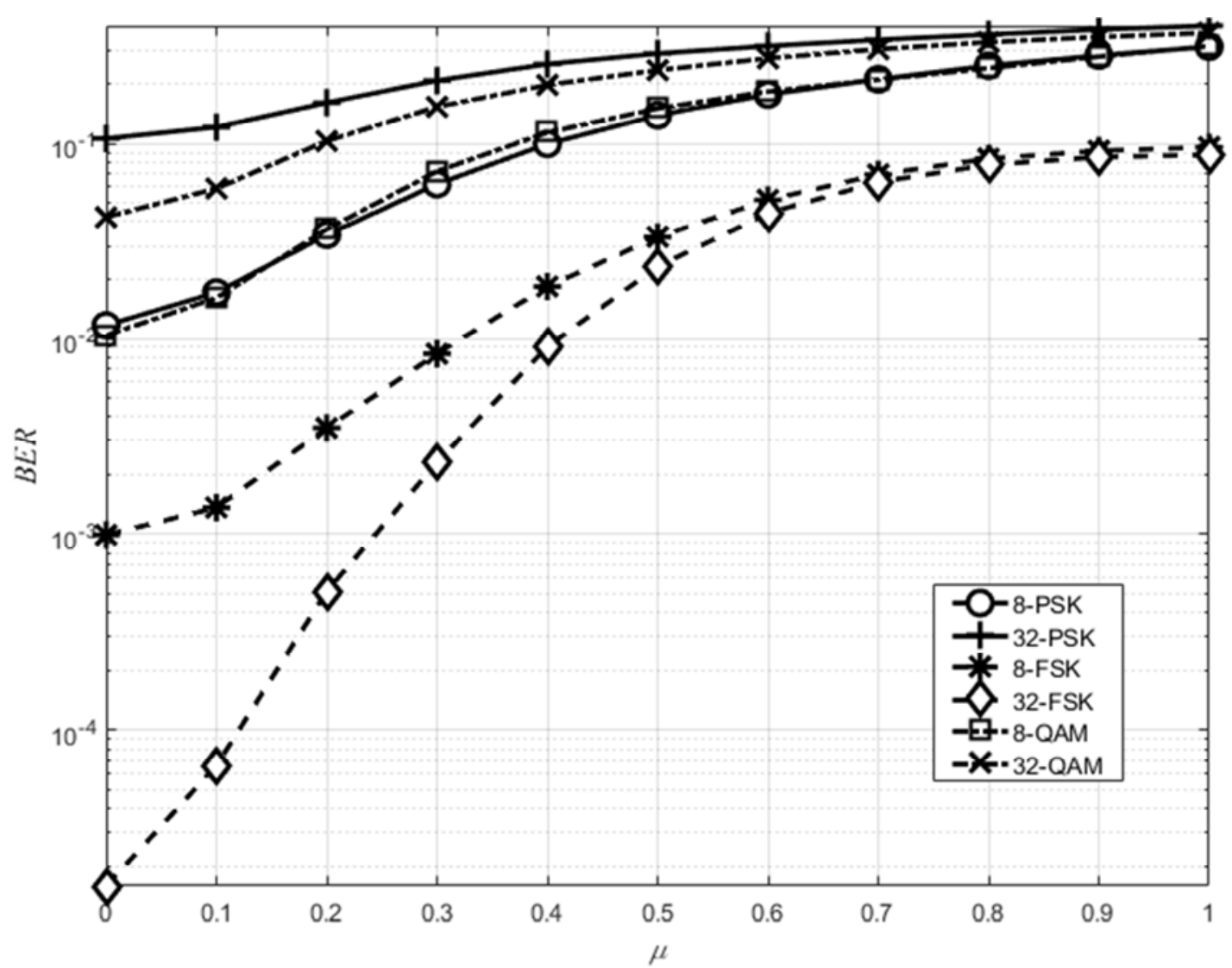

Figure 8. Relationship between the error probability, BER, in a two-beam channel and the intensity, $\mu$, of the reflected beam for different signal formats.

In Figure 7, the relationships between the error probability, BER, of receiving a binary symbol and the number of beams, $N$, propagating in a radio channel are shown for different types of modulation. It is noticeable that the multipath effect for some types of modulation leads to a sharp deterioration of this noise immunity index by 2-3 orders of magnitude.

Figure 8 shows the relationship between the error probability, BER, and the reflection coefficient, $\mu$, in the twobeam channel for different signal formats. It can be seen that with an increase in this index, the probability of an error increases by 2-3 orders of magnitude. The most stable, as shown by the simulation, are signals of the M-FSK format.

\section{Conclusions}

The influence of various parameters of the radio channel of communication with UAV on the quality of digital information transmission using different types of modulation (M-PSK, M-QAM, M-FSK) is studied in the paper. To obtain the results, the computer simulation method was used. A multipath propagation model with the number of beams up to 10 is used. The dependencies of the probability of erroneous reception of information on the parameters of the communication channel are obtained: the signal-to-noise ratio, the number and intensity of the propagation paths. It is shown that at a high intensity of the reflected beams $\mu>0.3$ and their number $N>3$, the quality of communication is reduced greatly. The energy parameters of the radio channel are calculated and the optimal frequency range is estimated from the maximum signal-to-noise ratio. It is indicated that the best in this criterion is the operating frequency range of 2-5 GHz. Calculations showed that the transmission coefficient of the radio channel is unstable and varies greatly in frequency. When using narrow-band signals M-PSK, M-QAM, this can lead to a situation where the signal can get into the "bad" frequency band and the quality of information transfer will decrease significantly. In connection with this, a radio channel with multiposition frequency modulation M-FSK was studied, when the transmission of discrete information is carried out using multiple parcels with different frequencies falling into both "bad" and "good" frequency zones. The results of the study showed that the probability of error in some cases improves by times compared to M-PSK and MQAM. This allows us to speak about the prospectivity of the format of the M-FSK. Taking into account the good performance of the latter format, further studies are planned to be carried out in the direction of use of M-FSK format signals and their prospective version of M-CPFSK (multi-position frequency modulated signals with a continuous phase) in the considered radio channel with different modulation indices and positioning, in particular CPFSK with modulation indices of 0.5 and 0.75 together with MIMO and OFDM systems [2127]. The obtained results can be used in the design of modern and promising UAV-to-ground communication systems. 


\section{References}

[1] Reg Austin, "Unmanned Aircraft Systems: UAVS Design, Development and Deployment"; John Wiley \& Sons Ltd, 2010, 372 p., ISBN: 978-0-470-05819-0.

[2] Y. Okumura, E. Ohmori, T. Kawano, and K. Fukuda, "Field strength and its variability in VHF and UHF land mobile radio service", Rev. Inst. Elec. Eng., 1968, V. 16, №9-10, pp. 825873.

[3] Hata M., "Empirical formula for propagation loss in land mobile radio service", IEEE Trans. Veh. Technol., 1980, v. VT-29, no. 3, pp. 317-325.

[4] Yuvraj Singh. "Comparison of Okumura, Hata and COST-231 Models onthe Basis of Path Loss and Signal Strength", International Journal of Computer Applications, 2012, Vol. 59, No. 11, December, pp. 37-41.

[5] Rafhael Amorim, Huan Nguyen, Preben Mogensen, "Radio Channel Modeling for UAV Communication Over Cellular Networks", IEEE Wireless Communications Letters. Volume: 6, Issue: 4, Aug. 2017.

[6] D. W. Matolak, R. Sun, "Air-ground channel characterization for unmanned aircraft systems: The hilly suburban environment", Proc. IEEE 80th Veh. Technol. Conf. (VTC Fall), pp. 1-5, Sep. 2014.

[7] D. W. Matolak, R. Sun, "Air-ground channel characterization for unmanned aircraft systems: The near-urban environment", Proc. IEEE Mil. Commun. Conf. (MILCOM), pp. 1656-1660, Oct. 2015.

[8] D. W. Matolak and R. Sun, "Unmanned aircraft systems: airground channel characterization for future applications", IEEE Veh. Technol. Mag., vol. 10, no. 2, pp. 79-85, Jun. 2015.

[9] Q. Feng, J. McGeehan, E. K. Tameh and A. R. Nix, "Path Loss Models for Air-to-Ground Radio Channels in Urban Environments", in Proc. IEEE Veh. Technol Conf. (VTCSpring'06), Melbourne, Australia, May 2006, pp. 2901-2905.

[10] Q. Feng, E. K. Tameh, A. R. Nix and J. McGeehan, "Modelling the Likelihood of Line-of-Sight for Air-to-Ground Radio Propagation in Urban Environments", in Proc. IEEE Global Commun. Conf. (GLOBECOM'06), San Francisco, USA, Dec. 2006, pp. 1-5.

[11] A. Al-Hourani, S. Kandeepan and A. Jamalipour, "Modeling Air-to-Ground Path Loss for Low Altitude Platforms in Urban Environments", in Proc. IEEE Global Commun. Conf. (GLOBECOM'14), Austin, USA, Dec. 2014, pp. 2898-2904.

[12] D. W. Matolak and R. Sun, "Air-Ground Channel Characterization for Unmanned Aircraft Systems-Part I: Methods Measurements and Models for Over-Water Settings", IEEE Trans. Veh. Technol., vol. 66, no. 1, pp. 26-44, 2017.

[13] R. Sun and D. W. Matolak, "Air-Ground Channel Characterization for Unmanned Aircraft Systems-Part II: Hilly and Mountainous Settings", IEEE Trans. Veh. Technol., vol. 66, no. 3, pp. 1913-1925, 2017.
[14] D. W. Matolak and R. Sun, "Air-Ground Channel Characterization for Unmanned Aircraft Systems-Part III: The Suburban and Near-Urban Environments", IEEE Trans. Veh. Technol., vol. 66, no. 8, pp. 6607-6618, 2017.

[15] R. Sun and D. W. Matolak, "Air-Ground Channel Characterization for Unmanned Aircraft Systems Part-IV: Airframe Shadowing", IEEE Trans. Veh. Technol., vol. 66, no. 9, pp. 7643-7652, 2017.

[16] Li Bing, "Study on Modeling of Communication Channel of UAV", Journal Procedia Computer Science, Volume 107, Issue C, April 2017, pp. 550-557.

[17] Zhihong Qiu, Xi Chu, Cesar Calvo-Ramirez, César Briso, and Xue-feng Yin, "Low Altitude UAV Air-to-Ground Channel Measurement and Modeling in Semiurban Environments", Wireless Communications and Mobile Computing, Volume 2017.

[18] İsmet Çuhadar and Mahir Dursun, "Unmanned Air Vehicle System's Data Links", Journal of Automation and Control Engineering, Vol. 4, No. 3, June 2016, pp. 189 -193.

[19] V. M. Sineglazov, A. O. Kuzmenko, "UAV communication link design", Electronics and Control Systems, 2015, no. 4 (46), pp. 72-76

[20] Boev N. M., "Methods of increasing spectral and energy efficiency in digital communication systems with unmanned aerial vehicles", Proceedings of MIPT, Moscow, Russia, 2014, V. 6, № 2, pp. 162-166.

[21] Kulikov G., Tambovskiy S., Belousov O., Shebakpolskiy M., "Advanced Methods of Signal Processing in Communication Systems with Unmanned Aerial Vehicles", Proceedings - The Second International Conference on Engineering and Telecommunication En\&T-2015, Moscow, Russia, 2015, pp. $38-40$.

[22] Kulikov G. V., "Interference immunity of receivers of modulated signals with a continuous phase in the presence of non-fluctuation interference", Radiotechnika, Moscow, Russia, 2003, №7, pp. 21-27.

[23] Kulikov G. V., Belousov O. B., "Multi-H CPM Signal Format Selection for Communication Systems with Complex Jamming Environment", Proceedings - 2014 International Conference on Engineering and Telecommunication, En\&T2014, Moscow, Russia, 2014, pp. 43-45.

[24] Seybold J. S., "Introduction to RF propagation", Willy Interscience Inc., 2005, 352 p., ISBN: 978-0-471-65596-1.

[25] Sklar B., "Digital Communications: Fundamentals \& Applications", 2 Edition; Pearson Education, 2009, 1164 p., ISBN: $9788131720929,8131720926$.

[26] Z. Wu, H. Kumar, and A. Davari, "Performance evaluation of OFDM transmission in UAV wireless communication," Proceedings of the thirty-seventh southeastern symposium on system theory, pp. 6-10, 2005.

[27] J. Chen, B. Daneshrad and W. Zhu, "MIMO Performance Evaluation for Airborne Wireless Communication Systems," inProc. IEEE Mil. Commun. Conf. (MILCOM'11), Baltimore, USA, Nov. 2011, pp. 1827-1832. 